\title{
ALIENACIÓN PARENTAL A LA LUZ DE LA LEGISLACIÓN DEL ESTADO DE TABASCO, MÉXICO
}

\section{PARENTAL ALIENATION IN LIGHT OF THE LEGISLATION OF THE STATE OF TABASCO, MEXICO}

Isi Verónica Lara-Andrade ${ }^{1}$ (D); Genny Guadalupe Mora-Fonz ${ }^{2}$ (D) ; Viviana Castellanos-Suárez ${ }^{3}$ (D).

1. Universidad Juárez Autónoma de Tabasco División Académica de Ciencias Sociales y Humanidades, México. isis.lara1234@gmail.com

2. Universidad Juárez Autónoma de Tabasco. División Académica de Ciencias Sociales y Humanidades, México. gennyfonz@hotmail.com

3. Universidad Juárez Autónoma de Tabasco. División Académica de Ciencias Sociales y Humanidades, México. vivihermosa_70@hotmail.com

*Correspondencia del Autor: Isi Verónica Lara Andrade, correo electrónico: isis.lara1234@gmail.com.

\section{RESUMEN}

La Alienación Parental (AP) es una forma sutil de violencia que se da sobre todo con la separación o el divorcio. Desacreditación y rechazo de forma abierta o encubierta al hablar o actuar de una manera descalificante o destructiva del otro progenitor. Mientras que el Síndrome de Alienación Parental (SAP) se ha entendido como conducta de denigración o rechazo por parte del niño a un progenitor, producto de la influencia maliciosa de alguno de sus padres o incluso de terceros. El Código Civil del Estado de Tabasco, menciona en tres ocasiones la noción "alienación parental", no como síndrome, sino a la acepción más amplia, sin que coloque a las niñas, niños y adolescentes en estado de indefensión y vulnerabilidad previendo que los padres desarrollen conductas que vayan en detrimento del o los infantes.

Palabras clave: Alienación Parental (AP); Síndrome de Alienación Parental (SAP); interés superior; maltrato infantil.

Cómo citar:

Lara-Andrade, Isi Verónica; Mora-Fonz, Genny Guadalupe; Castellanos-Suárez, Viviana. (2020). Alienación parental a la luz de la legislación del estado de Tabasco, México. Revista de Investigaciones Universidad del Quindio, 32(2), 77-87. https://doi.org/10.33975/riuq.vol32n2.457 


\begin{abstract}
Parental Alienation (PA) is a subtle form of violence that occurs mostly within separations or divorces; it is a type of discredit and rejection, openly or covertly, when speaking or acting in a disqualifying or destructive manner of the other parent. While Parental Alienation Syndrome (PAS) has been understood as a denigration or rejection behavior on the part of the child to a parent, product of the malicious influence of one parent or even third parties. The Civil Code of the State of Tabasco mentions in three occasions the notion of "parental alienation", not as a syndrome, but in its broadest meaning, in which it places children and teenagers in a state of helplessness and vulnerability, foreseeing that parents will develop behaviors that are detrimental to the infant(s).
\end{abstract}

Keywords: Parental Alienation (PA); Parental Alienation Syndrome (PAS); higher Interest; child abuse.

\section{INTRODUCCIÓN Y ANTECEDENTES}

En el presente artículo abordaremos sobre el tema de "alienación parental", término que tiene más de una década de haber sido introducido, sin embargo hoy en día es utilizado y argumentado con mayor intensidad en el ámbito judicial, por operadores jurídicos, abogados litigantes, profesionales de la salud mental, gobernados, en asuntos en los que se ven involucrados de manera indirecta e indirecta "menores" ante la separación de sus padres, y donde de manera ineludible la autoridad judicial tendrá que discernir (en caso de controversia) quien es el más apto de ellos para obtener la guarda y custodia y en su caso la patria potestad, por tanto su estudio se ha visto obligado desde un punto de vista psicológico y judicial.

Ante ello tenemos que el llamado Síndrome de Alienación Parental (SAP) tiene sus orígenes en los estudios de Richard A. Gardner, en 1985, donde categoriza una serie de conductas que los hijos toman en contra de uno de los padres, ya sea que censuren, critiquen o rechacen. Este concepto tiene componentes del llamado "lavado de cerebros" que implica la actuación de uno de los padres (alienante) que va contribuyendo al rechazo del otro (Bolaños, 2000).
En el ámbito científico no existe un consenso en cuanto al llamado SAP, ya que aún no existen suficientes investigaciones que permitan clasificar esa práctica como una enfermedad o síndrome de manera oficial (Organización Panamericana de la Salud, 2020). Sin embargo, es innegable que las consecuencias de esta práctica documentada tengan efectos en la salud mental del menor que se encuentra en medio de un problema de separación matrimonial o de concubinato.

Pese a que el concepto no tiene un sustento científico, en México y varios países está regulado. En el caso de México, la mayoría de las entidades federativas tiene en sus códigos civiles una serie de supuestos en las que la Alienación Parental aplica, dejando entrever una diferenciación entre el concepto de Síndrome (SAP) y Alienación Parental (AP).

El uso que se le da a este concepto en los litigios de orden familiar es cotidiano y esto ha generado a lo largo de años unos intensos debates que hablan de la vigencia o derogación de esta figura. En los debates no solo está vigente la cientificidad del término, sino la correlación que hay entre los derechos humanos con este concepto, en particular aquellos derechos como el derecho a la familia, derecho a la identidad, derecho a la 
justicia, derecho a ser escuchado (Rodríguez, 2011), lo anterior tomando en cuenta que en su aplicación colisionan dichos derechos, es decir se enfrenta uno de frente de otro, y siendo que todos merecen la misma atención al encontrarse en el mismo nivel de jerarquización, luego entonces deben ser estrictamente ponderados ya que de lo contrario el principio rector que rige cuando intervienen menores "interés superior del menor" se vería irreparablemente dañado.

Se afirma lo anterior, pues al momento que se da la separación entre el padre e hijo, imperan los derechos del menor a relacionarse con ambos padres mediante la guarda, custodia $\mathrm{y}$ convivencias con el padre no custodio que -en su caso-, determine la autoridad.

De lo anterior es de advertir que en cualquier decisión que se tome -aun cuando también se encuentren inmersos derechos de los padres-, se debe de considerar como eje rector, el principio superior del menor, y el derecho a tener una convivencia sana del menor con sus padres, es derecho preponderante del niño (LPDNNyA, Artículo 24), y solo en casos sumamente excepcionales y debidamente justificadas puede suspenderse esta convivencia. Sirven de apoyo diversos criterios que se localizan bajo los rubros siguientes: sirve de apoyo el criterio interpretativo que se localiza bajo el rubro:" INTERÉS SUPERIOR DEL MENOR. SU FUNCIÓN NORMATIVA COMO PRINCIPIO JURÍDICO PROTECTOR", "INTERÉS SUPERIOR DEL NIÑO. ES UN PRINCIPIO DE RANGO CONSTITUCIONAL IMPLÍCITO EN LA REGULACIÓN DE LOS DERECHOS DE LOS MENORES PREVISTOS EN EL ARTÍCULO $4^{\circ}$ CONSTITUCIONAL", "INTERÉS SUPERIOR DEL NIÑO. FUNCIÓN EN EL ÁMBITO JURISDICCIONAL".

\section{METODOLOGÍA}

Se aborda el tema desde el punto de vista jurídico, puesto que es ahí donde se debate sobre su existencia y efectos jurídicos, identificándolo en la Codificación Civil del Estado de Tabasco, la forma de como conceptualiza, dicha figura jurídica y los parámetros y/o mecanismos que existen para no ser motivo de vulneración a los derechos de los niños, puesto que la falta de su ponderación ocasionaría daños irreparables como veremos más adelante.

En esa tesitura, para comprender las dimensiones complejas del término se podría dividir su estudio entre una parte teórica-comparativa y el estudio en los ordenamientos jurídicos.

\section{MARCO TEÓRICO Y COMPARATIVO}

La expresión "Síndrome de Alienación Parental (SAP)" fue publicada por primera vez por el norteamericano, experto en psiquiatría infantil y forense, Gardner, como se señala en párrafos anteriores; quien realizaba peritajes judiciales cuando formuló el concepto, publicándolo en un artículo titulado: Recent Trends in Divorce and Custody Litigation, en el cual señala que el síndrome de alienación parental es un trastorno infantil que surge casi exclusivamente en el contexto de las disputas por la custodia de los niños. Su manifestación primaria es la campaña de denigración del niño contra un padre; una campaña que no tiene justificación, resultado de la combinación de una programación (lavado de cerebro) del adoctrinamiento parental. (Gardner, 1985; 2005). En este caso el Síndrome de Alienación Parental es un conjunto de fenómenos que ocurren cuando hay conflictos (Silva, 2021) en la familia y uno de los progenitores aliena a un menor sobre el otro progenitor, alienar viene del latín alienare (sacar, afuera), así se aprecian un conjunto de afectaciones en los menores en torno a las relaciones entre padres e hijos.

Como signos o síntomas del síndrome principalmente comienza por un rechazo que llega hasta el odio patológico e injustificado hacia el progenitor alienado, lo que lleva al menor a consecuencias devastadoras en su desarrollo físico y psicológico, en este proceso se van sumando acciones que desvirtúan los vínculos 
entre padres e hijos, el objetivo final es retirar de su vida diaria y la de los hijos al progenitor objeto de la alienación (Gardner, 2005). La expresión síndrome de alienación parental particularmente fue utilizada en el ámbito judicial, donde se fue instalando entre los psicólogos, abogados, peritos y funcionarios de la justicia. Su utilización se multiplicó exponencialmente y fue avalado por un gran número de profesionales (Alemán, 2020).

Por su parte, la Alienación Parental (AP) es una forma sutil de violencia que se da sobre todo después de la separación o el divorcio, el proceso de desacreditación y rechazo se va dando de forma abierta o encubierta al hablar o actuar de una manera descalificante o destructiva del otro progenitor, con la clara intención de alejar o indisponer al hijo o a los hijos contra el otro progenitor y con ello romper el vínculo.

Mientras el Síndrome de Alienación Parental (SAP) se ha entendido como conducta de denigración o rechazo de su progenitor por parte del niño, producto de la influencia maliciosa de alguno de sus padres o incluso de terceros. Es un proceso en el que se manipula la mente de los menores para que repitan mentiras, historias fantasiosas, descripciones de abusos o maltratos inexistentes, creados por el padre o la figura que aliena al menor.

La distinción entre los dos es que la alienación parental (AP) se centra en cómo se comporta el padre alienante con los hijos y el padre alienado, mientras que el Síndrome de Alienación Parental (SAP), se centra más en los efectos que dicha conducta tiene en los hijos alienados. La Alienación Parental (AP) es "cualquier tipo de conducta, ya sea consciente o inconsciente, que podría alterar la relación entre un niño $\mathrm{y}$ el otro padre". Esta definición es diferente del síndrome de alienación parental (SAP), que se define como "una alteración provocada por uno de los progenitores en la que los niños denigran y critican a uno de los padres de forma injustificada y / o exagerada, resultado de una combinación entre un adoctrinamiento (lavado de cerebro) de uno de los padres y las propias contribuciones del niño a la difamación del padre alienado." (Quinde, 2020).

La alienación parental (AP) es el rechazo persistente del niño al contacto con uno de sus dos progenitores, a quien profesa una hostilidad injustificadamente, mientras que establece una alianza con el otro, no presentando un conjunto especificos de síntomas necesariamente. En el caso de la alineación parental, no existe consenso respecto a la necesaria concurrencia de estrategias de alineación por parte de uno de los progenitores, puesto que podría darse que un menor presentase rechazo a relacionarse con el otro en ausencia de conductas obstaculizadoras; siendo la alienación, en este caso, el resultado de la resolución de un conflicto de lealtades provocadas por la discordia interparental. Por lo cual el SAP puede ser considerado, un subtipo específico de alienación parental (AP), (González, 2015).

En el campo doctrinal, autores como el psicólogo José María Bouza, señalan que "es un desorden que surge habitualmente en el contexto de las disputas por la custodia de un hijo", pero matizan que la presencia de la alienación parental no se da por la disputa de la tenencia, sino por el interés específico de uno de los progenitores de adjudicarse la pertenencia total de los hijos, física y afectiva, como una posesión, como un objeto, a no compartir. Es habitual que la alienación parental resulte ser más sencillo ser entendida por los profesionales del Derecho que por los de la salud mental, porque se detecta en el ámbito Judicial, en la rutina del seguimiento de los expedientes y como tal debemos considerar a la alienación parental como una "Patología Jurídica” (Ruíz, 2011).

Elestudio del SAPseharealizado primordialmente desde disciplinas del área de la salud, en especial desde la psicología. Los estudios de Gardner abordan una serie de características para detectar la alienación parental, este describe "síntomas primarios" que aparecen en niños afectados por el SAP de acuerdo a Bolaños (2017): 
1. Campaña de denigración. Se nota la obsesión del niño en odiar a uno de sus padres.

2. No hay una justificación clara para el desprecio.

3. No son capaces de encontrar algún punto positivo en los padres odiados.

4. El niño piensa que no ha sido influenciado por nadie.

5. Muestra indiferencia total por los sentimientos del padre odiado

6. Los argumentos parecen ensayados

7. Animadversión a la familia extensa y red social del progenitor odiado.

"Estas características han despertado una serie de cuestionamientos, por un lado, los argumentos que consideran que esos elementos son subjetivos para categorizar el SAP y, por el otro, los argumentos que no apoyan el uso de estos elementos en asuntos judiciales ya que pueden incluso ser dañinos para los menores, así como reforzar estereotipos de género, en especial en los juicios donde la custodia la tiene la madre" (Bolaños, 2017, p. 245). Los argumentos esenciales de los detractores del SAP es que su fundamento se ubica en la ideología patriarcal y machista, y que en ningún momento las actitudes de repulsa hacia el padre son consecuencia de actos generados por la madre, sino que lo que en realidad ocurre es que en algunos casos en que no se otorga la guarda y custodia compartida, los(as) menores adoptan actitudes de resistencia hacia uno de los padres cuyo origen es muy diverso.

En correlación con lo anterior, se han documentado en asuntos judiciales en los Estados Unidos que, como "tratamiento" a este problema, se decide que la custodia del niño o niña sea retirada del padre "alienante" y se traspase al padre o madre que el niño rechaza, los estudios arrojan que esto puede tener consecuencias en la salud mental del menor (Mercer, 2019).

Lo anterior es así porque el menor ya se encuentra con un entorno familiar establecido, donde se desarrolla emocional y físicamente, y al darse el desprendimiento de dicho entorno en el que él menor se considera se encuentra en óptimas condiciones y bajo el cuidado del progenitor "bueno", tal situación provocaría daños psicoemocionales y menoscabaría su desarrollo integral al considerar encontrarse ahora del lado del padre -a decir de el- "malo".

En México, los criterios se han ido utilizando para la elaboración de algunos protocolos de actuación que buscan generar la mayor objetividad para tratar estos asuntos, al menos estableciendo 9 puntos para identificar la AP (PJEC, 2018):

1. Campañas de injurias y desaprobación.

2. Explicaciones triviales para justificar la campaña de desacreditación.

3. Ausencia de ambivalencia en su odio al progenitor alienado.

4. Extensión del odio al entorno del progenitor alienado.

5. Ausencia de sentimientos de culpa y defensa del progenitor alienador

6. Escenarios prestados. Presencia de escenas $\mathrm{y}$ actos que el hijo adopta como propios cuando jamás estuvo presente.

7. Fenómeno de pensador independiente

8. Dificultades al momento de las visitas

9. Inmersión judicial. El progenitor alienador tiende a abusar de los procesos judiciales y la supervisión de las visitas.

En la actualidad el síndrome de alienación parental es considerado como un tipo de maltrato/ castigo infantil en donde se presentan situaciones en las que algún progenitor obstaculiza las relaciones de sus hijos con el otro progenitor, de tal forma que si se busca en los libros de psicopatología psiquiátrica o psicológica el síndrome de alienación parental no consta en el Manual Diagnóstico y Estadístico de los Trastornos Mentales DSM-5 libro de diagnóstico clínico utilizado por el área de la salud mental, concretamente en el capítulo 19 se ubica dentro se los problemas de relación y familiares y se encontrará esta práctica como una forma sutil y casi invisible de maltrato infantil que produce 
un gran daño en el bienestar emocional y en el desarrollo de los niños, niñas y adolescentes que lo sufren, ya que dicho proceso alienante está destinado a romper el vínculo de los hijos con uno de sus progenitores a partir de inventar historias de abusos y maltratos, incluso sexuales, que nunca existieron, influenciados principalmente por sus madres, abuelas, familiares y profesionales relacionados con los procesos de separación.

Por tanto, al existir dichos actos de alienación que se evidencian en las conductas previamente citadas, y las cuales pueden ser advertidas por expertos en la materia, así como que los menores involucrados son los que realmente se ven dañados emocionalmente ante las conductas intencionadas por el progenitor alienador en busca de la ruptura del vínculo afectivo hacia el otro progenitor, ante ello es obligación del Estado - a través de la autoridad correspondiente - como garante de los derechos fundamentales del menor, priorizar el estado emocional y la integridad personal de éste por encima de cualquier conflicto de orden familiar que se llegue a suscitar, y salvaguardando al menor de maltrato infantil, por tanto los padres y el Estado deben velar por ello, la manipulación infantil existe y es considerada maltrato infantil y por tanto requiere atención inmediata.

La Magistrada Graciela G. Buchanan Ortega, en su ensayo sobre su trascendencia en el ámbito judicial "alienación parental", recomienda que desde el momento que sea denunciada o identificada en un asunto del orden familiar la existencia de la alienación parental, aun cuando ésta sea catalogada como ligera, en aras del cumplimiento a la obligación contraída por el Estado para con los infantes y que estos obtengan un desarrollo adecuado a su edad y condición, así como que éstos siempre se vean protegidos en su estabilidad física y mental, en la sentencia correspondiente se haga un estudio del maltrato detectado, apoyándose en los dictámenes periciales ofrecidos por las partes, las evaluaciones psicológicas y de trabajo social, en general de todo el material probatorio que se pueda allegar (Buchanan, 2012).

\section{MARCO JURÍDICO}

El marco jurídico relativo a este concepto engloba tres categorías importantes: la legislación civil vigente; los criterios de derechos humanos (Silva \& Martínez, 2019) de orden internacional; las resoluciones y jurisprudencia del poder judicial. En lo relativo a la legislación civil del Estado de Tabasco, el Código Civil menciona en tres ocasiones la noción "alienación parental":

Artículo 265: Medidas en favor de los hijos.

En los procedimientos judiciales de divorcio, la autoridad que conozca de aquéllos debe tomar todas las medidas necesarias para realizar el interés que el Estado tiene, conforme a los artículos 405 y 406, en relación con los hijos de los cónyuges que sean menores o sólo estén concebidos.

En tanto se decrete el divorcio y posterior a éste, los padres evitarán cualquier acto de presión o manipulación hacia los hijos, encaminado a destruir los vínculos afectivos con el padre o la madre.

Durante el procedimiento, el juez podrá allegarse de los elementos de convicción necesarios para determinar los alcances de su sentencia, considerando la situación económica de los divorciantes, la valoración psicológica de ambos padres o de los hijos, debiendo escuchar a los padres y a los hijos, según resulte necesario, para prevenir y evitar conductas de violencia familiar o alienación parental, considerando el interés superior de la niñez

Artículo 281. Protección de los hijos o cónyuges inocentes

Quienes ejerzan la patria potestad deben permitir el acercamiento constante de los menores con sus ascendientes, salvo causa justificada y por razones de seguridad de 
los menores. En consecuencia, evitarán cualquier acto de alienación parental.

Artículo 405.- Atención del ser humano.

Es de orden público la atención del ser humano durante la gestación, su nacimiento y su minoría de edad.

Al momento de pronunciarse en relación con la situación de menores en caso de derecho familiar, el juzgador podrá dictar, de ser necesario, medidas sobre alienación parental; entendida ésta como la presión, manipulación o inducción que realizan el padre o la madre hacia los menores para predisponerlos negativamente contra uno u otra, según sea el caso.

Sin que lo anterior pueda dar lugar a estimar que su inclusión en nuestra legislación civil local coloque a las niñas, niños y adolescentes en estado de indefensión y vulnerabilidad, si partimos que el propósito de dicha propuesta normativa fue precisamente salvaguardar el pleno desarrollo de los niños, niñas y evitar que los padres desarrollen conductas que vayan en detrimento del o los infantes. 1

1 Cfr. El Considerando Tercero del Decreto 290, publicado en el Suplemento 7648 K del Periódico Oficial del Estado de Tabasco de 23 de diciembre de 2015, en el que, si bien se alude al síndrome de alienación parental, se establece de manera reiterada que los motivos que subyacen a la modificación normativa es la de salvaguardar el pleno desarrollo de los niños y niñas. Concluyendo que, garantizar un pleno desarrollo integral del menor para una vida digna es una obligación que como Estado debemos realizar, mediante la implementación de mecanismos e instrumentos efectivos, que permitan garantizar la protección, goce y disfrute de los derechos humanos de las niñas y niños, conforme lo establecen los diferentes Tratados Internacionales suscritos por el Estado Mexicano en aras de velar y proteger los derechos fundamentales del menor, de igual forma como lo indica nuestra Constitución Nacional, así como la Ley General de los Derechos de Niñas, Niños y Adolescentes en su artículo 13. Por tales consideraciones se estima viable la propuesta
Por tanto, la connotación de dicho fenómeno en la legislación sustantiva civil local no se limita al denominado "Síndrome", sino a la acepción más amplia, esto es, aquella en la que su abordaje puede verse de manera multidisciplinaria y siempre atendiendo el interés superior de los menores, de ahí que sea válida.

Máxime que, no existe disposición alguna en el referido ordenamiento que condicione la pérdida de la patria potestad ni tampoco se utiliza como limitante para la escucha de los menores. Por el contrario, se insiste en que la inclusión de tal figura privilegia el interés superior de los menores, en congruencia con lo previsto en los artículos 8.1 y 9.3 de la Convención sobre los Derechos del Niño, que textualmente señalan:

Artículo 8. 1 los Estados Parte se comprometen a respetar el derecho del niño a preservar su identidad, incluidos la nacionalidad, el nombre y las relaciones familiares de conformidad con la ley $\sin$ injerencias ilícitas.

Artículo 9.3 Los Estados Parte respetarán el derecho del niño que esté separado de uno o de ambos padres a mantener relaciones personales y contacto directo con ambos padres de modo regular, salvo si ello es contrario al interés superior del niño.

Tal línea de pensamiento es congruente con la determinación adoptada por la Suprema Corte de Justicia de la Nación, al resolver la Acción de Inconstitucionalidad 11/2016, en la que después de analizar doctrina existente sobre el tema, determinó las notas distintivas entre el referido Síndrome de Alienación Parental y la sola alienación, señalando la existencia de esta última desde antes del descubrimiento del aludido Síndrome, la que incluso es conocida

de modificación al Código Civil con el objeto de establecer medidas para salvaguardar el interés superior del menor y evitar que los padres desarrollen conductas de alienación parental que vayan en detrimento del o los infantes. 
bajo diversas denotaciones. 2

Así también al resolver la Acción de Inconstitucional 120/2017, analiza los alcances - de la norma impugnada -, al ejercer alienación parental sobre el menor por alguno de los progenitores, esto es "la suspensión del ejercicio de la patria potestad" y determina declarar su invalidez, por considerar que su aplicación se prevé en forma irrestricta,- entre otros- sin permitir la ponderación judicial en torno a la idoneidad, necesidad y eficacia de la norma en el caso concreto para salvaguardar el interés superior del menor.

Por tanto, al retomar nuestro Código Civil vigente en el Estado, el cual como ya se dijo 2 El Pleno de la Suprema Corte de Justicia de la Nación, al resolver la referida Acción de Inconstitucionalidad interpuesta en contra de disposiciones del Código Civil del Estado de Oaxaca, que contemplaban lo relativo a la alienación parental, no soslayó la falta de consenso científico existente, aun así determinó que conforme al artículo $4^{\mathrm{o}}$ de la Constitución Federal y al artículo 19 de la Convención sobre los Derechos del Niño, no está en duda que el legislador tiene el deber de establecer un sistema normativo apropiado y eficaz para garantizar el derecho de los menores de edad a una vida libre de violencia; por tanto, si las conductas identificadas como "alienación parental" entrañan una injerencia que puede afectar la integridad psico-emocional de los menores, ese riesgo de daño, válidamente justifica su regulación, no obstante la incertidumbre científica que pudiere prevalecer en torno a dicho concepto. Sobre todo, no debe desatenderse que, aun cuando no exista uniformidad o consenso entre las teorías y opiniones del foro de la psicología sobre la conceptualización de la denominada alienación parental y su forma de detección o diagnóstico; lo relevante es que, el legislador, conforme a su función, asignó un contenido específico a la conducta que se propuso regular, y en ese sentido, con independencia de que para la comprensión del fenómeno se hubiere apoyado en las opiniones y los aportes de las ciencias que estudian la conducta humana, lo cierto es que, para efectos de la legislación civil, el legislador hizo la descripción de lo que se entendería por "alienación parental", a efecto de entender actualizada la hipótesis legal. no condiciona la pérdida de la patria potestad ni tampoco se utiliza como limitante para la escucha de los menores, vemos que se le otorga la discrecionalidad al juzgador de aplicar las medidas que considere necesarias, (según su idoneidad, necesidad y eficacia) y según sea el caso para salvaguardar el derecho de los menores, así también salvaguarda su derecho de ser escuchado, y de allegarse de todos los medios probatorios necesarios entre ellos las valoraciones psicológicas para determinar los alcances de su sentencia, en la que forzosamente, atendiendo el numeral 508 del Código de Procedimientos Civiles en vigor en el Estado, el cual establece -entre otras cosas-, que en los juicios de divorcio se resolverá de oficio lo relativo al cuidado de los hijos y patria potestad. Dicho de otra manera el juzgador ante un caso concreto, se ve obligado - a fin de resolver el conflicto-, a la aplicación del principio rector del interés superior del menor, a través de un proceso de ponderación de los derechos del menor tales como el derecho a ser protegido contra toda forma de violencia, exigencia del artículo 4 constitucional, así como el artículo 19 de la Convención sobre los Derechos del niño, Ley General de los Derechos de las Niñas, Niños y Adolecentes en su artículo 13 fracción VII y VIII, el derecho del menor a expresar su opinión, previsto en el artículo 12 de la convención sobre los derechos del niño, el derecho a vivir en familia y a mantener relaciones con sus progenitores artículo 8, 9 ,19, de la Convención sobre los derechos del niño" pues al verse lesionados éstos derechos humanos del menor se requiere de un análisis de optimización del derecho humano, es decir, que derecho "pesa" más que el otro, pues forzosamente deberá de restringir uno.

También es importante el uso de protocolos de actuación para la atención de estos casos complejos, uno de ellos puede ser el Protocolo de Actuación para quienes imparten Justicia en los casos que involucren Niñas, Niños y Adolescentes, el cual, si bien no trata el asunto de la alienación parental de manera directa, si es una buena guía para interpretar los derechos 
humanos.

Ahora bien, la SCJN ha dictado diversas resoluciones que van ampliando el estudio de la alienación parental, predominando hasta ahora el criterio de que el problema constitucional no es el concepto por ahora, sino los supuestos y medidas que se aplican en torno al mismo.

Tabla 1. Resoluciones del Poder Judicial de la Federación

\begin{tabular}{|c|c|}
\hline Resoluciones PJF & Extractos de la resolución \\
\hline $\begin{array}{l}\text { Acción de inconstitucionalidad 120/2017 } \\
\text { Promovió: Comisión Estatal de los Derechos } \\
\text { Humanos de Baja California } \\
\text { Se resolvió el } 12 \text { de noviembre de } 2019 \text {. }\end{array}$ & $\begin{array}{l}\text { Se considera como inconstitucional el hecho } \\
\text { de que, con motivo de la alienación parental, } \\
\text { se pueda ordenar la suspensión o pérdida de } \\
\text { la patria potestad -desproporcionalidad de } \\
\text { las medidas-, y no así, que el legislador haya } \\
\text { regulado tal fenómeno y las conductas que, en } \\
\text { su caso, lo generan }\end{array}$ \\
\hline $\begin{array}{l}\text { Acción de Inconstitucionalidad 111/2016 } \\
\text { Promueve: CNDH } \\
\text { Se resuelve el } 14 \text { de noviembre de } 2019 \text {. }\end{array}$ & $\begin{array}{l}\text { Se consideran inconstitucionales el artículo } \\
178 \text { del Código Penal del Estado de Michoacán } \\
\text { "Se considera como violencia familiar la } \\
\text { alienación parental demostrada, respecto de } \\
\text { sus hijos o adoptados". }\end{array}$ \\
\hline $\begin{array}{l}\text { Acción de Inconstitucionalidad 11/2016 } \\
\text { Promueve: Defensoría de los Derechos } \\
\text { Humanos del Pueblo de Oaxaca } \\
\text { Se resuelve el } 24 \text { de octubre de } 2017\end{array}$ & $\begin{array}{l}\text { Considera inconstitucional el hecho de que, } \\
\text { con motivo de la alienación parental, se ordene } \\
\text { la suspensión o perdida de la patria potestad. }\end{array}$ \\
\hline $\begin{array}{l}\text { Tesis aislada publicada el } 22 \text { de enero de } 2021 . \\
\text { Tesis: VII.2o.C.239 C (10a.) } \\
\text { Prueba psicológica practicada a un menor } \\
\text { de edad. previo a acordar la realización de } \\
\text { una segunda, con la intención de descartar la } \\
\text { existencia de alienación parental, el juzgador } \\
\text { debe agotar todos los procesos alternos, atento } \\
\text { al interés superior del menor }\end{array}$ & $\begin{array}{l}\text { Este Tribunal Colegiado considera que previo a } \\
\text { ordenar una segunda intervención psicológica } \\
\text { a un menor de edad, el juzgador debe agotar } \\
\text { todos los procesos alternos que deriven del } \\
\text { caso concreto, atento al interés superior del } \\
\text { menor, como encomendar a un especialista } \\
\text { el análisis de los registros ya existentes } \\
\text { a efecto de conducir la prueba pericial o } \\
\text { analizar la pertinencia de la repetición, o un } \\
\text { segundo interrogatorio o pericial al menor de } \\
\text { edad a la luz de diverso material probatorio } \\
\text { para determinar si es necesario el desahogo } \\
\text { de una diversa pericial, en principio, sin la } \\
\text { intervención del menor. }\end{array}$ \\
\hline
\end{tabular}




\section{SÍNTESIS}

1. Aun cuando las críticas hacia la regulación del concepto Alienación Parental están justificadas en cuanto a su clasificación científica, por el momento, las discusiones sobre la inconstitucionalidad solo están enfocadas en los supuestos de la perdida de la patria potestad, la cual se ha considerado desproporcionada en los supuestos en que se aplica.

2. Para profundizar los estudios, deben de realizarse análisis interdisciplinarios desde expertos en la materia.

3. Es necesario el uso de protocolos y criterios internacionales de derechos humanos.

4. Constatar, como premisa básica, que el obstaculizar el derecho del o la menor a mantener sus vínculos emocionales y afectos con ambos progenitores y familiares por igual, es una forma de maltrato emocional que puede ocasionarles un daño a su bienestar y desarrollo emocional.

5. Ser consciente que la posible existencia de casos de manipulación de un progenitor en contra de sus hijas e hijos para lograr que éstos(as) rechacen y muestren sentimientos de repulsa hacia el otro, es una situación que puede, y de hecho así ocurre, ser ocasionada por un cónyuge en contra del otro, pero no necesariamente de la madre en contra del padre.

6. En Tabasco si bien se ha utilizado la figura de Alienación Parental en las resoluciones judiciales, ha sido en el sentido del concepto genérico de la definición propia de la palabra alienar, hacer perder, extraño a uno y ajeno, no como un Síndrome (SAP), es decir se enfoca a la conducta, ya sea consciente o inconsciente, que podría alterar la relación entre un niño y el otro padre.

\section{CONCLUSIÓN}

Es innegable que en muchas ocasiones cuando existe la separación de padres se ve afectado de forma directa "el menor", esto es desde el primer momento que no llegan a un acuerdo en quien de los dos progenitores se ha de quedar con la guarda y custodia del hijo, por tanto, es menester acudir al órgano jurisdiccional e iniciar un procedimiento, a fin de que sea la autoridad quien decida por que los padres no pudieron hacerlo.

En la mayoría de los casos ese acuerdo se hace imposible porque uno de ellos no desea separarse del otro, por lo que ello origina sentimientos de rencor, coraje, odio, hacía el otro cónyuge o pareja, así también dicha separación origina la necesidad de saber quien se ha de quedar con los hijos, (guarda y custodia) y por lo tanto el derecho de los alimentos de éstos. Motivos por los cuales desean conseguir a los hijos como meros trofeos, y como "medios" para obtener sus objetivos, y es ahí, a donde se presenta la "alienación parental" daño que si bien pudiera considerar algo muy subjetivo y difícil de probar.

Sin embargo ello no significa que no exista, que no esté en la mayoría de los asuntos del orden familiar, empero nuestra legislación al contemplar dicha figura, otorga facultades y mecanismos a los jueces, para poder prevenirla, $o$ en su caso erradicarla, no siendo un camino fácil, pues requiere de la verdadera sensibilización del juzgador, y de un análisis del caso concreto que se le presente, advertir si existe la colisión de derechos humanos y su optimización y justificar a través de verdaderos argumentos (sustentados) la decisión que ha de tomar al respecto. 


\section{REFERENCIAS}

1. Alemán, J. A. (2020). La alienación parental, presente en familias del noroeste de México. Los nuevos retos del Trabajo Social. Cuadernos de Trabajo Social, 33(2), 349-361.

2. Bolaños Cartuijo, Ignacio. (2000). Estudio descriptivo del síndrome de alienación parental en procesos de separación y divorcio. Diseño de aplicación de un programa piloto de mediación familiar, tesis doctoral, Universidad Autónoma de Barcelona, España.

3. Bolaños, Iñaki. (2017). El Síndrome de Alienación Parental. Descripción y Abordajes Psico-legales, Psicopatología, Clínica, Legal y Forense en Montoya López, Adolfo Eduardo, \& Rivas Duarte, Jaqueline, "La Alienación Parental y su regulación en México, una omisión en su legislación". Revista del Posgrado en Derecho de la UNAM, 4(7), 242-243.

4. Buchanan Ortega Graciela G. (2012). Alienación Parental, Ensayo sobre su trascendencia en el ámbito judicial. Primera Edición.

5. Diario Oficial de la Federación. (2000). Ley para la Protección de los Derechos de Niñas, Niños y Adolescentes. México.

6. Gardner, R.A. (1985). Recent Trends in Divorce and Custody Litigation. Academy Forum, 29(2), 3-7.

7. Gardner, R.A. (2005). Basic Facts About the Parental Alienation Syndrome.

8. González Sarrió, Ignacio. (2015). Psicología jurídica y forense, Diferencia entre el Síndrome de Alienación Parental (SAP) y la Alineación Parental (AP).

9. Mercer, Jean. (2019). Examining Parental Alienation Treatments: Problems of Principles and Practices. Child and Adolescent Social Work Journal (36), 351-363.

10. Organización Panamericana de la Salud. (2020). Clasificación Internacional de Enfermedades (CIE).

11. Poder Judicial del Estado de Campeche. (2018). Protocolo de Actuación en casos de Alienación Parental en los procesos judiciales que involucran a niñas, niños y adolescentes.

12. Quinde, C., \& Frit, A. (2020). Incorporación del Síndrome de Alienación Parental como forma de Violencia Psicológica al menor en la Ley 30364.

13. Rodríguez Quintero, Lucía. (2011). Alienación parental y derechos humanos en el marco jurídico nacional. Algunas consideraciones, en ET AL, Alienación Parental, México, Comisión Nacional de Derechos Humanos.

14. Ruíz Carbonell, Ricardo. (2011). La llamada Alineación Parental: la experiencia en España, Alineación Parental, Comisión Nacional de Derechos Humanos.

15. Silva Hernández, F., \& Martínez Prats, G. (2019). La justicia alternativa como derecho humano. JURÍDICAS CUC, 15(1), 263-284. https://doi.org/10.17981/juridcuc.15.1.2019.10

16. Silva-Hernández, Francisca. (2020). Hacia una comprensión del conflicto en justicia alternativa. Revista de Investigaciones Universidad del Quindío, 32(1), 61-65. https://doi.org/10.33975/riuq. $\operatorname{vol} 32 \mathrm{n} 1.435$ 